\title{
BROWN TUMOR OF BONE AS INITIAL MANIFESTATION OF PRIMARY HYPERPARATHYROIDISM
}

\author{
Sergiy Cherenko \\ Chief of Endocrine Surgery Department \\ Ukrainian Scientific and Practical Center of Endocrine Surgery Ministry of Health of Ukraine \\ 13-a Klovsky descent, Kyiv, Ukraine, 01021 \\ sergmakar5@gmail.com \\ Victoriia Hoperiia \\ Head of Department of Endocrine Pathology \\ Ukrainian Scientific and Practical Center of Endocrine Surgery Ministry of Health of Ukraine \\ 13-a Klovsky descent, Kyiv, Ukraine, 01021 \\ drhoperia@mail.ru \\ Hennadii Bandura \\ Researcher, Department of Endocrine Surgery \\ Ukrainian Scientific and Practical Center of Endocrine Surgery Ministry of Health of Ukraine \\ 13-a Klovsky descent, Kyiv, Ukraine, 01021 \\ doc.bandura@gmail.com
}

\begin{abstract}
Primary hyperparathyroidism (PHPT) is characterized with primary hyperproduction of parathyroid hormone (PTH) by affected parathyroid glands and with a corresponding increase in concentration of extracellular calcium. Long-term development of PHPT through decades leads to calcium-related symptoms and distinct changes in the skeleton, kidneys, digestive tract and other organs. In the clinical picture of PHPT, granulomatous pseudo-tumors in bones are observed as a rare manifestation of the disease - these lesions, which could imitate malignancy, are called «brown tumors». The present publication that goes into details about a clinical diagnosis, in which a brown tumor became the primary manifestation of PHPT, may be useful for physicians across various fields.
\end{abstract}

Keywords: primary hyperparathyroidism; brown tumors; diagnosis; clinical manifestation.

\section{Introduction}

Primary hyperparathyroidism (PHPT) is characterized by primary hyperproduction of parathyroid hormone (PTH) and by a corresponding elevation of extracellular calcium level. PTH acts to increase the concentration of calcium in extracellular fluid by triggering the release of calcium and phosphates from the bone matrix, renal calcium reabsorption and mediated by Vitamin D3 enhanced digestive absorption of calcium. Long-term development of PHPT through decades leads to distinct (and sometimes irreversible) changes in the skeleton, kidneys, biliary tract and other organs. In developed countries, the predominant way of PHPT discovering is the screening of elevated blood calcium level; thus, new cases of PHPT are largely reported as mild or asymptomatic forms. Establishing the diagnosis of PHPT is essentially a laboratory task, and actually it requires only two parameters to match: the elevated levels of serum calcium and serum PTH. The clinical diagnosis of PHPT is complicated by the lack of specific symptoms; such symptoms as susceptibility to fractures, thirst, bone pain, urinary and biliary stones indicate a late stage of the disease. In the clinical picture of PHPT granulomatous pseudo-tumors in bones are observed as a rare manifestation of the disease. These lesions, which are called «brown tumors», are a consequence of excessive bone resorption and remodeling, which are directly mediated by PHT. Brown tumors are more common in patients with secondary hyperparathyroidism related to renal failure, in which the level of PTH sometimes reaches $1000-3000 \mathrm{pg} / \mathrm{mL}$; but they may appear in severe cases of PHPT as well [1]. Radiological signs of brown tumors are characterized by deformation of long bones (femur, tibia, shoulder) or spongy bones (ribs, collarbone, hip bones), accompanied by bone 
erosion or tumor-like bone formations [2]. In practice, many cases of bone "brown" tumorssimulate malignancy, and doctors rarely suspect PHPT initially [3]. Even the elevated level of serum calcium accompanied by a bone tumor is more likely to be perceived by a clinician as a paraneoplastic or metastatic hypercalcemia, rather than brown tumor appearance of severe long standing PHPT [4]. To differentiate metastatic bone lesions from parathyroid carcinoma and brown tumor arising in presence of parathyroid adenoma positronemission to mography with fluoro-2deoxy-D-glucose (FDG) was proposed; authors found intensive FDG uptake in brown tumor but not in parathyroid adenoma [5]. There are very few published examples that offer an illustrated and well documented case of brown bone tumor in a patient with PHPT, so we consider this study to bea useful and interesting medical information for doctors of different profiles (endocrinologists, oncologists, surgeons, trauma orthopedists, radiologists).

\section{Case Report}

Woman, 28, became ill in August 2015. Clinical symptoms consisted of general weakness, nausea, lack of appetite, thirst (about 5 liters of liquid consumed per day), frequent urination (4-5 times per night). A family doctorsupposed the diagnosis of chronic gastritis exacerbation, and the relevant treatment lasted two months. No positive dynamic was observed; conversely, an additional symptoms of pain in the bones of spine and lower limbs appeared. Pain killers brought no relief. Because of the leg and back pain, the patient started to limp on her left leg. A source of pain and a swelling were revealed in the upper third of the left tibia. Topical treatment was introduced; after the radiography, a radiologist suspected a myeloma. The patient was sent for consultation to a hematologist-oncologist, who in turn directed her to National Cancer Institute, Kyiv, Ukraine. In the Institute, she was examined by a hematologist-oncologist and an orthopedic oncologist. On December 24, 2015 X-ray examination of the skeleton was performed. The X-ray of the tibia is shown on Fig. 1.

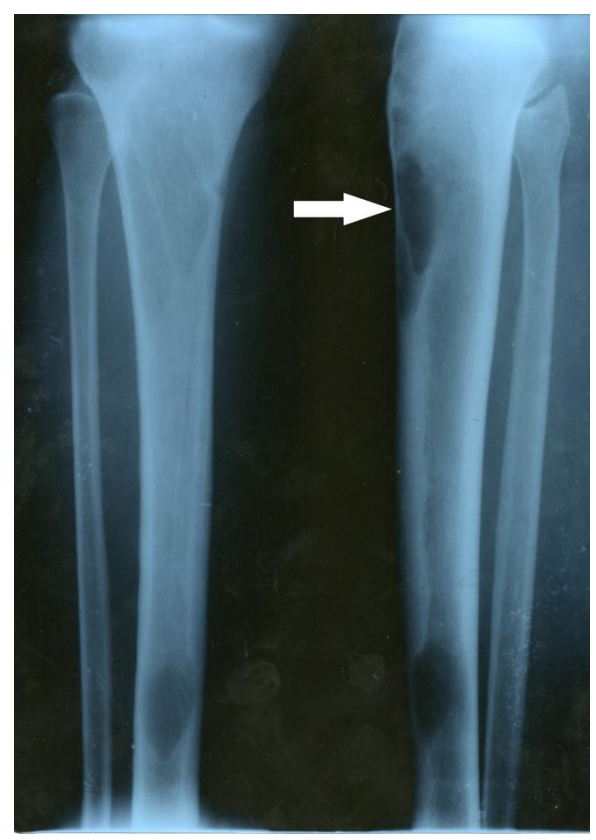

Fig. 1. Radiography of Lower Limb Bones: Brown Tumor in Left Tibia

The hematologist-oncologist assigned needle biopsy of bone marrow, and the conclusion was obtained on January 11, 2016: no bone marrow lesions were observed. Core biopsy of tumor in the tibia was additionally performed, which on January 19, 2016 yielded the following result: the biopsied material contain fibroplastic tissue with angiomatosis, microhemorrhages, and multinucleated giant cells of osteoclastictype, that are arranged in clusters and laid separately. Given the clinical data (multifocal lesions), a brown tumor related to hyperparathyroidism was suspected. It 
was eventually recommended to determine the level of parathyroid hormone and ionized calcium (the uniqueness of the situation is that a pathologist has been the first to diagnose PHPT!). The patient turned for consultation to Ukrainian Scientific and Practical Center for Endocrine Surgery, Endocrine Organs and Tissue Transplantation. The patient presented the results of examinations performed in National Cancer Institute, as well as the results of laboratory analyses from January 28, 2016: PTH - $1064 \mathrm{pg} / \mathrm{mL}$ (the normal level is $15-65 \mathrm{pg} / \mathrm{mL}$ ); calcium total $-3.28 \mathrm{mmol} / \mathrm{L}$ (norm.: 2.2-2.55 mmol/L); calcium ionized - $1.94 \mathrm{mmol} / \mathrm{L}$ (norm.: 1.05-1.30 mmol/L); phosphorus $-0.54 \mathrm{mmol} / \mathrm{L}$ (norm.: 0.81-1.45 mmol/L); vitamin D $(25-\mathrm{OH})-5.1 \mathrm{ng} / \mathrm{mL}$ (norm.: $30-100 \mathrm{ng} / \mathrm{mL}$ ). In the clinical conditions, the patient went through the standard examination. Ultrasonography (US) of the neck was performed February 09, 2016, which revealed a hypoechoic structure, sized $25 \times 24 \mathrm{~mm}$ attached to the lower pole of the left thyroid lobe. Thyroid gland is normal. The US examinations of internal organs and urinary system have not identified any abnormalities. The thyroid function was not compromised and the level of thyroid stimulating hormone (TSH) was $1.23 \mathrm{mIU} / \mathrm{L}$ (norm.: 0.5-3.5 mIU/L). Dual-energy X-ray absorbtiometry (DXA) revealed osteopenic syndrome (Total body bone density: BMD $-0.968 \mathrm{~g} / \mathrm{cm}^{3}$, T-score: -2.0 , Z-score: -2.6 ). The patient was prescribed 3000 IU of Ergocalciferol per day and scheduled for surgery. After the short-term preparation in the surgical ward, the operation was performed on February 19, 2016: bilateral neck revision from Kocher incision; removal of adenoma of the left lower parathyroid gland; selective central neck dissection; lymphadenectomy. Intraoperative frozen section biopsy confirmed the removal of parathyroid adenoma (Fig. 2), 35×25 mm large and $6350 \mathrm{mg}$ in weight; lymph nodes of the central neck compartmentdidn’t contain metastases.

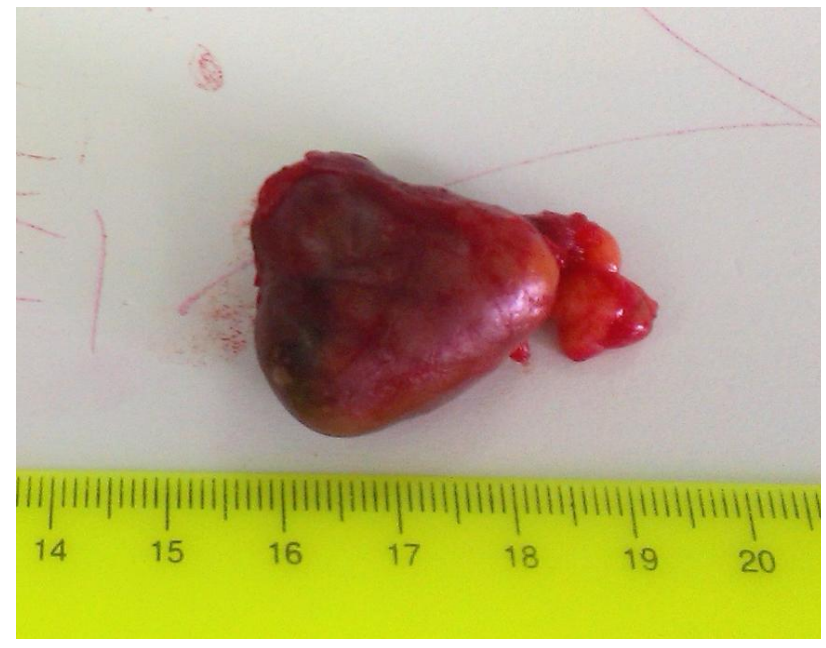

Fig. 2. Adenoma of the Parathyroid Gland

An hour after the surgery the PTH level dropped to $36.7 \mathrm{pg} / \mathrm{mL}$ (norm.: $15-65 \mathrm{pg} / \mathrm{mL}$ ); the decrease in ionized calcium was not so significant $-1.86 \mathrm{mmol} / \mathrm{L}$ (norm.: $1.05-1.30 \mathrm{mmol} / \mathrm{L}$ ). Clinical signs of hypocalcemia were recorded only 3 days after the surgery, and a significant decreasing of ionized calcium was observed at $6^{\text {th }}$ dayafter the surgery $(1.20 \mathrm{mmol} / \mathrm{L})$, while the patient was receiving $2 \mu \mathrm{g}$ of Alfacalcidol and $2000 \mathrm{mg}$ of calcium per day. The patient also continued to take Ergocalciferolduring recommended 2 months course. The patient was discharged in a satisfactory condition; follow-up checks were scheduled to control the levels of PTH, calcium, phosphorus, and vitamin D at 3, 6 and 12 months. Also, the schedule included a control DXA examination in 12 months, and radiography of the lower limb bones in 6 and 12 months.

\section{Pathological Examenation}

Inour Department of Pathology was examined histological material obtained by the core biopsy of brown tumor of the tibia and parathyroidectomy. 
Pathological examination of the biopsied tibia tumor fragment revealed numerous giant cells surrounded by fusiform stromal cells. In the stroma, extensive areas of hemorrhage were defined [6, 7]. Histopathological diagnosis: brown tumor related to hyperparathyroidism (Fig. 3).

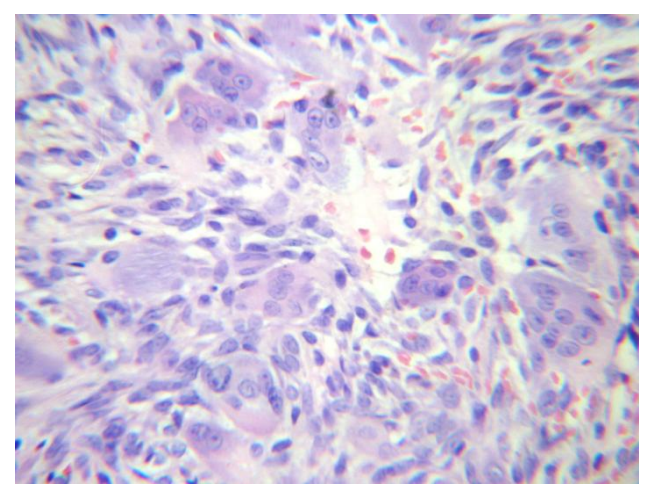

Fig. 3. Brown Tumor Related To Hyperparathyroidism. Numerous giant cells surrounded by fusiform stromal cells. Hematoxylin and eosin stain, $\times 400$

Histopathological examination of parathyroid tumor revealed its mostly solid structure, with defined zone of trabecular structure. The tumor was built from parathyroid chief cells with centrally located hyperchromic, monomorphic nuclei and faintly eosinophilic cytoplasm. The tumor was surrounded by a thin capsule of connective tissue. A normal parathyroid gland tissue was located around the tumor. Histopathological diagnosis: adenoma of parathyroid gland (Fig. 4).

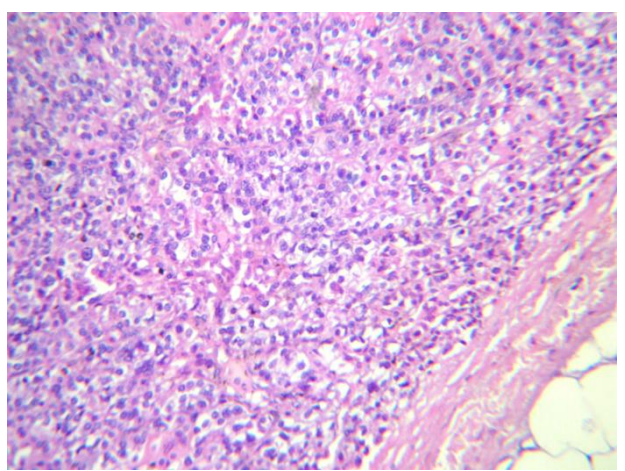

Fig. 4. Adenoma of Parathyroid gland.Hematoxylin and eosin stain; $\times 200$

\section{Discussion}

Primary hyperparathyroidism is one of the most common diseases of the endocrine system, with 3-5 occurrences per 1,000 in the population (and up to $3 \%$ among women aged over 60 ). On the other hand, distinct clinical features are being found in PHPT patients to a smaller extent, and true diagnosis is often delayed when serum calcium is not screened. Only by increasing the overall awareness among physicians of such a widespread disease as PHPT we might prevent unnecessary and dangerous treatments, such as resection or biopsy of bones that contain pseudo-tumors. A laboratory analysis, in which high blood calcium level coincide with high level of PTH, is a compelling argument for low probability of a malignant bone lesion with accompanying hypercalcemia: in that case, the PTH level is always reduced. Differential diagnosis of brown tumor related to hyperparathyroidism includes giant cell tumor, reparative granuloma, and bone aneurysms [8]. Some authors connect probability of severe bone involvement during the course of PHPT with vitamin D deficiency [9]. Presented clinical case might be crucially related with prominent vitamin D deficiency. Biochemically proven PHPT diagnosis andradiological signs of brown tumor generally abandon theneed in core biopsy of altered bone. A full regression of brown tumors is observed within 1-3 years after recovery from PHPT [10]. 


\section{Conclusions}

Universal screening of hypercalcemiais essential for timely diagnosis of PHPT. Educational program that could raise awareness among physicians about bone manifestations of hyperparathyroidism, such as brown tumors, allows reducing diagnostic and treatment errors.

\section{Refferences}

[1] Van Herden, J. A., Beahrs, O. H., Woolner, L. B. (1977). The pathology and surgical management of primary Hyperparathyroidism. SurgClin North Am., 57 (3), 557-563.

[2] Vera, L., Dolcino, M., Mora, M., Oddo, S., Gualco, M., Minuto, F.,Giusti, M. (2011). Primary hyperparathyroidism diagnosed after surgical ablation of a costal mass mistaken for giant-cell bone tumor: a case report. Journal of Medical Case Reports, 5 (1), 596. doi: 10.1186/1752-1947-5-596

[3] Joyce, J. M., Idea, R. J., Grossman, S. J., Liss, R. G., Lyons, J. B. (1994). Multiple Brown Tumors in Unsuspected Primary Hyperparathyroidism Mimicking Metastatic Disease on Radiograph and Bone Scan. Clinical Nuclear Medicine, 19 (7), 630-635. doi: 10.1097/00003072-199407000-00016

[4] Kalambokis, G., Economou, G., Kamina, S.,Papachristou, D. J., Bai, M.,Tsianos, E. V. (2005). Multiple brown tumors of the ribs simulating malignancy. Journal of Endocrinological Investigation, 28 (10), 738-740. doi: 10.1007/bf03347558

[5] Hsu, C.-H., Liew, P.-L., Wang, W., Leung, T.-K., Yang, K.-M. (2008). Enhanced FDG Uptake in Brown Tumors Mimics Multiple Skeletal Metastases in a Patient with Primary Hyperparathyroidism. ActaRadiol, 49 (8), 949-950. doi: 10.1080/02841850802314788

[6] Aoki, J., Moriya, K., Yamashita, K., Fujioka, F., Ishii, K., Karakida, O.et. al (1991). Giant Cell Tumors of Bone Containing Large Amounts of Hemosiderin. Journal of Computer Assisted Tomography, 15 (6), 1024-1027. doi: 10.1097/00004728-199111000-00023

[7] Matsushige, T., Nakaoka, M., Yahara, K., Kagawa, K., Miura, H., Ohnuma, H., Kurisu, K. (2008). Giant cell tumor of the temporal bone with intratumoral hemorrhage. Journal of Clinical Neuroscience, 15 (8), 923-927. doi: 10.1016/j.jocn.2007.03.013

[8] Haque, A. U., Moatasim,A.(2008). Giant cell tumor of bone: a neoplasm or a reactivecondition? Int J Clin Exp Pathol, 1 (6), 489-501.

[9] Silverberg, S. J. (2007). Vitamin D Deficiency and Primary Hyperparathyroidism. Journal of Bone and Mineral Research, 22 (S2), V100-V104. doi: 10.1359/jbmr.07s202

[10] Agarwal, G., Mishra, S. K., Kar, D. K., Singh, A. K., Arya, V., Gupta, S. K.,Mithal, A. (2002). Recovery pattern of patients with osteitis fibrosa cystica in primary hyperparathyroidism after successful parathyroidectomy. Surgery, 132(6), 1075-1085. doi: 10.1067/msy.2002.128484 